\title{
Sheared rotation effects on kinetic stability in enhanced confinement tokamak plasmas, and nonlinear dynamics of fluctuations and flows in axisymmetric plasmas (Revised March 12, 1998)
}

\author{
G. Rewoldt, M. A. Beer, M. S. Chance, T. S. Hahm, Z. Lin, and W. M. Tang \\ Plasma Physics Laboratory, Princeton University, \\ Princeton, New Jersey $08543-0451$
}

\begin{abstract}
Sheared rotation dynamics are widely believed to have significant influence on experimentally-observed confinement transitions in advanced operating modes in major tokamak experiments, such as the Tokamak Fusion Test Reactor (TFTR) [D. J. Grove and D. M. Meade, Nucl. Fusion 25, 1167 (1985)], with reversed magnetic shear regions in the plasma interior. The high- $n$ toroidal drift modes destabilized by the combined effects of ion temperature gradients and trapped particles in toroidal geometry can be strongly affected by radially-sheared toroidal and poloidal plasma rotation. In previous work with the FULL linear microinstability code, a simplified rotation model including only toroidal rotation was employed, and results were obtained. Here, a more complete rotation model, that includes contributions from toroidal and poloidal rotation and the ion pressure gradient to the total radial electric field, is used for a proper self-consistent treatment of this key problem. Relevant advanced operating mode cases for TFTR are presented. In addition, the complementary problem of the dynamics of fluctuation-driven $\mathbf{E} \times \mathbf{B}$ flow is investigated by an integrated program of gyrokinetic simulation in annulus
\end{abstract}


geometry and gyrofluid simulation in flux tube geometry.

$52.35 \mathrm{Qz}, 52.65 \mathrm{Tt}, 52.55 \mathrm{Fa}$

Typeset using REVTEX 


\section{INTRODUCTION}

Sheared rotation dynamics are widely believed to have significant influence on experimentally-observed confinement transitions in advanced operating modes in major tokamak experiments, such as the Tokamak Fusion Test Reactor ${ }^{1}$ (TFTR), with reversed magnetic shear regions in the plasma interior. ${ }^{2}$ The high-n (toroidal mode number) toroidal drift modes destabilized by the combined effects of ion temperature gradients and trapped particles in toroidal geometry can be strongly affected by radially-sheared toroidal and poloidal plasma rotation. In previous work with the FULL linear microinstability code, ${ }^{3,4}$ a simplified rotation mode ${ }^{5,6}$ including only toroidal rotation was employed, and results ${ }^{7,8}$ were obtained. However, a more complete rotation model, ${ }^{9}$ that includes contributions from toroidal rotation and poloidal rotation and the ion pressure gradient to the total radial electric field, is needed for a proper self-consistent linear treatment of this key problem. Specifically, this new model is capable of including effects of the $\mathbf{E} \times \mathbf{B}$ shearing frequency in general geometry, ${ }^{10}$ written in terms of equilibrium quantities, and evaluated at the outboard midplane, $\omega_{E}=2 \pi\left|\left[\left(R B_{\theta}\right)^{2} / B\right] \partial / \partial \Psi\left(E_{r} / R B_{\theta}\right)\right|$, where $\Psi$ is the poloidal flux and $B_{\theta}$ is measured via the Motional Stark Effect diagnostic, ${ }^{2}$ and $k_{r} \simeq k_{\theta}$ has been assumed in accordance with the results from measurements in TFTR ${ }^{11}$ and simulations. ${ }^{12-15}$ We include $\omega_{E}$ on an equal basis with the other rotation model terms in the linear instability calculation. Both rotation models result in stabilizing or destabilizing drifts in addition to the usual magnetic drifts. All of the rotation terms are now evaluated for a numerically-calculated flux-coordinate magnetohydrodynamic (MHD) equilibrium with magnetic surfaces of arbitrary cross-sectional shape and aspect ratio. Needed $\mathbf{E} \times \mathbf{B}$ levels for linear stabilization with this approach are compared with corresponding levels from the heuristic stabilization criterion $\omega_{E} \sim \gamma_{0}$, where $\gamma_{0}$ is the linear growth rate without rotation effects, as observed in ion temperature gradient (ITG) mode simulations, ${ }^{14,16}$ which has sometimes shown good correlations with experimental transitions. Relevant advanced operating mode cases for TFTR will be presented. 
Many magnetic confinement experiments have indicated that $\mathbf{E} \times \mathbf{B}$ shear can suppress turbulence and consequently lead to significant reduction of plasma transport. ${ }^{17,18}$ It has been also observed in gyrofluid simulations that the fluctuation driven $\mathbf{E} \times \mathbf{B}$ flow plays a dominant role in the nonlinear saturation of ITG turbulence (both with and without trappedelectron dynamics ${ }^{15}$ ) which has been identified as a likely deterrent to efficient confinement in

tokamak plasmas. It is therefore of vital importance for nonlinear simulations to accurately treat the dynamics of the fluctuation driven flow. This complementary nonlinear problem is investigated in the present work by an integrated program of gyrokinetic simulation in annulus geometry ${ }^{19}$ and gyrofluid simulation in flux tube geometry.

The new linear rotation model implemented in the FULL code is worked out in Sec. II, and results for several TFTR cases are presented and compared in Sec. III. In Sec. IV, results from three dimensional global gyrokinetic simulations are used to investigate the validity regimes of estimates of poloidal rotation damping and the residual level. Results for the dynamics of turbulence-driven fluctuating $\mathbf{E} \times \mathbf{B}$ flows from nonlinear flux-tube gyrofluid simulations are presented and discussed in Sec. V. Conclusions are given in Sec. VI.

\section{LINEAR ROTATION MODEL}

We will implement a linearized version of the rotation model described in Ref. 9, and solve the corresponding gyrokinetic equation by an extension of the method described in Ref. 3. While it was shown in Refs. 20 and 21 that the ballooning representation ${ }^{22}$ breaks down for substantial values of the Mach number, it was also shown in Refs. 20 and 23 that this representation is still usable for small values of the Mach number, and we will thus continue to employ it here. A prescription for the ballooning parameter ${ }^{22} \theta_{0}$ is needed, in addition to the rotation model itself. The simplest choice, $\theta_{0}=0$, which is the usual choice in the absence of rotation, is employed here. An alternative would be to average the eigenfrequency over $0 \leq \theta_{0} \leq 2 \pi$, as specified in Ref. 21. However, a better prescription could in principle be determined as follows: One-dimensional (ballooning representation) 
and two-dimensional calculations for toroidal drift modes have been compared for the older rotation model of Refs. 5 and 6 in Ref. 8, and a way of modeling one of the missing twodimensional effects in the one-dimensional calculation was found there. This was the effect of 'eigenfunction shearing,' in which the individual eddies in the two-dimensional eigenfunction twist as the Mach number increases, and the value of $k_{r}$ at $\theta=0$ increases. By making $\theta_{0}$ (which enters the ballooning representation expression for $k_{r}$ ) an explicit, fitted function of the local Mach number, reasonable quantitative agreement was obtained for the growth rates between the one-dimensional and two-dimensional calculations. This procedure is not carried out for the new rotation model presented in this section, but in principal it could be. A related comparison was made in Ref. 24 .

We employ the PEST-I flux coordinate system described in more detail in Ref. 3, in which $\zeta$ is the toroidal angle, $\theta$ is a poloidal angle variable, and $\psi$ is a radial coordinate related to the poloidal flux $\Psi$ by $d \Psi=2 \pi B_{0} \mathcal{F}(\psi) d \psi$, and $v=\left(R_{0} / 2 \pi\right) \int d^{3} x / R^{2}$ and also the functions $\mathcal{F}(\psi), g(\psi), q(\psi)$, and $p(\psi)$ are calculated as part of the MHD equilibrium solution. The toroidal major radius is denoted as $R$, the toroidal major radius at the magnetic axis is $R_{0}$, the magnetic field strength at the magnetic axis is $B_{0}$, and the Jacobian is $\mathcal{J}=v R^{2} / 2 \pi R_{0}$. Also, the collision operator discussed in Refs. 4 and 25 is used. The gyrocenter velocity is given by Eq. (15) of Ref. 9, but for the unperturbed orbit, to lowest order in $\rho_{j} / L$, where $\rho_{j} \equiv\left(2 T_{j} / m_{j}\right)^{1 / 2} /\left(e_{j} B_{0} / m_{j} c\right)$ is the gyroradius for species $j$ and $L$ is an equilibrium radial length scale, it becomes

$$
\frac{d \overline{\mathbf{R}}}{d t}=v_{\|} \mathbf{b}+\mathbf{u}_{E}+\frac{1}{e_{j} B} \mathbf{b} \times\left[\mu \nabla B+m_{j}\left(v_{\|} \mathbf{b}+\mathbf{u}_{E}\right) \cdot \nabla\left(v_{\|} \mathbf{b}+\mathbf{u}_{E}\right)\right] .
$$

Here, $\mathbf{b} \equiv \mathbf{B} / B, \mathbf{u}_{E} \equiv \mathbf{b} \times \nabla \Phi / B$, and we will neglect any poloidal angle dependence of the equilibrium electrostatic potential $\Phi$ to this order, for the reasons given in Ref. 9, so that $\Phi$ depends only on $\psi$. Note that in Eq. (1) there are both linear terms and quadratic terms with respect to $\mathbf{u}_{E}$, and that some of the terms are proportional to $\nabla \mathbf{u}_{E}$, and thus involve $\Phi^{\prime \prime}$ (and therefore $E_{r}^{\prime}$ ). In the Maxwellian equilibrium distribution function $F_{j}$, we include a parallel shift by $u_{\zeta j}$ (also referred to as $\left.V_{\phi}\right)$, so that $F_{j} \propto \exp \left(-E / T_{j}\right)$ and 
$\nabla \ln F_{j}=\nabla \ln n_{j}\left[1+\eta_{j}\left(E / T_{j}-3 / 2\right)\right]+\left(m_{j} / T_{j}\right)\left(v_{\|}-u_{\zeta}\right) \nabla u_{\zeta j}-\left(\mu / T_{j}\right) \nabla B$, where $E \equiv$ $\left(m_{j} / 2\right)\left(v_{\|}-u_{\zeta j}\right)^{2}+\mu B$. Then, after some algebra, the linearized gyrokinetic equation can be written in the ballooning representation in the form

$$
\begin{aligned}
\frac{\partial \hat{h}_{\sigma j}}{\partial t}+i \mathbf{k} \cdot \frac{d \overline{\mathbf{R}}}{d t} \hat{h}_{\sigma j}+\nu_{f j} \hat{h}_{\sigma j}= & -i\left\{\omega-\mathbf{k} \cdot \mathbf{u}_{E}-k_{\| \mid} u_{\zeta j}-\omega_{* j}\left[1+\eta_{j}\left(\frac{E}{T_{j}}-\frac{3}{2}\right)\right.\right. \\
& \left.\left.+\frac{m_{j}}{T_{j}} u_{\zeta j}\left(v_{\|}-u_{\zeta j}\right) \frac{r_{n j}}{r_{u \zeta j}}\right]\right\} \frac{e_{j}}{T_{j}} F_{j} \hat{\phi}(\theta) J_{0},
\end{aligned}
$$

in standard notation. Here, the hat $\left({ }^{\wedge}\right)$ indicates that a quantity has been decomposed in the ballooning representation; $\sigma \equiv \operatorname{sign}\left(v_{\|}\right), \hat{h}_{\sigma j} \equiv \hat{f}_{\sigma j}+e_{j} \hat{\phi}(\theta) F_{j} / T_{j}, f_{j}$ is the perturbed distribution function, the operator $\mathrm{k} \equiv-i \nabla$ is to be evaluated in the ballooning representation in the PEST-I flux coordinates, $r_{u \zeta j} \equiv-\left(d \ln u_{\zeta j} / d r\right)^{-1}, J_{0} \equiv J_{0}\left(k_{\perp} v_{\perp} / \Omega_{j}\right)=J_{0}(\theta)$, and the rest of the notation is standard. Equation (2) is written in the electrostatic limit, but the generalization to the electromagnetic case is straightforward, as shown in Refs. 3 and 4.

On both sides of Eq. (2), the quantity $\mathbf{k} \cdot \mathbf{u}_{E}$ appears. With the ballooning representation in our coordinate system, this becomes $\mathbf{k} \cdot \mathbf{u}_{E}=-n \Phi^{\prime}(\psi) / B_{0} \mathcal{F}(\psi)$, which can be Taylor expanded about $\psi^{(0)}$, the orbit time-average value of $\psi$, as $\mathbf{k} \cdot \mathbf{u}_{E} \simeq$ $\left(-n / B_{0}\right)\left[\Phi^{\prime}\left(\psi^{(0)}\right) / \mathcal{F}\left(\psi^{(0)}\right)+\left.\left(\psi-\psi^{(0)}\right)\left(\Phi^{\prime \prime} / \mathcal{F}-\Phi^{\prime} \mathcal{F}^{\prime} / \mathcal{F}^{2}\right)\right|_{\psi_{(0)}}\right]$. The first term in the Taylor series is the usual Doppler shift term, and the second term $\left[\propto(\partial / \partial \Psi)\left(E_{r} / R B_{\theta}\right)\right]$ contains the $\mathbf{E} \times \mathbf{B}$ shearing frequency, $\omega_{E}$. Thus, $\omega_{E}$ enters the linear calculation through this term in Eq. (2), and will therefore appear in the final mode equation. The constancy of the toroidal canonical angular momentum can be used to obtain a computationally useful relation for evaluating this second term, which is $\psi-\psi^{(0)} \simeq\left(m_{j} c / B_{0} \mathcal{F} e_{j}\right)\left[R v_{\|}-\left(R v_{\|}\right)^{(0)}\right]$.

The additional rotational terms on the left hand side of Eq. (2), other than the Doppler shift term, can be combined with the magnetic gradient and curvature drift terms to give an extended drift frequency. After considerable algebra, it can be evaluated in the PEST-I flux coordinates as:

$$
\begin{aligned}
\omega_{d j}(\theta)= & -\frac{2 \pi n c T_{j}}{\epsilon_{j}}\left[\left(\hat{v}^{2}+\hat{v}_{\|}^{2}\right)\left[\kappa_{1}+\left(\theta-\theta_{0}\right) \kappa_{2}\right]-\frac{\hat{v}_{\perp}^{2} B_{0}}{4 \pi \mathcal{F} B^{2}} \frac{d \beta}{d \psi}+\frac{1}{v_{j}^{2}}\left[\kappa_{3}+\left(\theta-\theta_{0}\right) \kappa_{4}\right]\right. \\
& \left.+\frac{\hat{v}_{\|}}{v_{j}^{2}}\left[\kappa_{5}+\left(\theta-\theta_{0}\right) \kappa_{6}\right]\right]-\frac{n m_{j} c}{B_{0}^{2} \mathcal{F} e_{j}}\left(\frac{\Phi^{\prime \prime}}{\mathcal{F}}-\frac{\Phi^{\prime} \mathcal{F}^{\prime}}{\mathcal{F}^{2}}\right)\left[R v_{\|}-\left(R v_{\|}\right)^{(0)}\right],
\end{aligned}
$$


where $v_{j} \equiv\left(2 T_{j} / m_{j}\right)^{1 / 2}, \hat{v} \equiv v / v_{j}, \hat{v}_{\|} \equiv v_{\|} / v_{j}, \hat{v}_{\perp} \equiv v_{\perp} / v_{j}, \hat{u}_{\zeta j} \equiv u_{\zeta j} / v_{j}$, and the six $\kappa^{\prime}$ 's can be expressed in terms of the PEST-I equilibrium and mapping metric quantities, as follows:

$$
\begin{aligned}
& \kappa_{1}=-\frac{1}{4 \pi B_{0} \mathcal{F} R^{2}}\left[\mathcal{F} \frac{\partial}{\partial \psi}\left(\frac{R^{2}}{\mathcal{F}}\right)+B_{0}^{2} \mathcal{F}^{2} \frac{\partial}{\partial \theta}\left(\frac{\nabla \psi \cdot \nabla \theta}{B^{2}}\right)-\frac{R^{2} B_{0}^{2}}{2 B^{2}} \frac{d \beta}{d \psi}\right], \\
& \kappa_{2}=\frac{1}{4 \pi B_{0} \mathcal{F} R^{2}}\left[B_{0}^{2} \mathcal{F}^{2} \frac{\partial}{\partial \theta}\left(\frac{4 \pi^{2} R^{2} q q^{\prime}}{v^{2} B^{2}}\right)\right], \\
& \kappa_{3}=\frac{2 B_{0} \mathcal{F}}{4 \pi B^{2}}\left[\frac{B^{2}}{B_{0}^{2} \mathcal{F}^{2}}\left(\frac{\partial}{\partial \psi} \frac{|\nabla \Phi|^{2}}{B^{2}}\right)+\left(\frac{\nabla \psi \cdot \nabla \theta}{R^{2}}\right)\left(\frac{\left(\Phi^{\prime}\right)^{2} g^{2} R_{0}^{2} B_{0}^{2}}{B^{4} R^{2}} \frac{\partial}{\partial \theta}|\nabla \psi|^{2}\right.\right. \\
& \left.\left.+\frac{\left(\Phi^{\prime}\right)^{2}|\nabla \psi|^{2}}{B^{2} R^{2}} \frac{\partial}{\partial \theta} R^{2}\right)\right]-\frac{2 \Phi^{\prime}}{B^{2} 2 \pi B_{0} \mathcal{F}}\left[\frac{p^{\prime} \Phi^{\prime}|\nabla \psi|^{2}}{B^{2}}+\frac{\Phi^{\prime}|\nabla \psi|^{2}}{R^{2}} \frac{\partial}{\partial \psi} R^{2}\right. \\
& \left.+\Phi^{\prime \prime}|\nabla \psi|^{2}+\Phi^{\prime}\left(\frac{\partial}{\partial \psi}|\nabla \psi|^{2}\right)+\Phi^{\prime} \nabla \psi \cdot \nabla \theta \frac{1}{R^{2}} \frac{\partial}{\partial \theta} R^{2}+\Phi^{\prime} \frac{\partial}{\partial \theta} \nabla \psi \cdot \nabla \theta\right], \\
& \kappa_{4}=\frac{2 B_{0} \mathcal{F}}{B^{2} 4 \pi}\left(-\frac{q^{\prime} R_{0} g}{\mathcal{J F}}\right)\left(\frac{\left(\Phi^{\prime}\right)^{2} g^{2} R_{0}^{2} B_{0}^{2}}{B^{4} R^{2}} \frac{\partial}{\partial \theta}|\nabla \psi|^{2}+\frac{\left(\Phi^{\prime}\right)^{2}|\nabla \psi|^{2}}{B^{2} R^{2}} \frac{\partial}{\partial \theta} R^{2}\right), \\
& \kappa_{5}=\frac{B B_{0} \mathcal{F} \Phi^{\prime}}{B^{2} \mathcal{J} \pi}\left[\frac{q}{B^{2}} \frac{\partial}{\partial \theta} \nabla \psi \cdot \nabla \theta-\frac{q}{B^{4} R^{2}} \nabla \psi \cdot \nabla \theta\left(B_{0}^{2} \mathcal{F}^{2} \frac{\partial}{\partial \theta}|\nabla \psi|^{2}\right.\right. \\
& \left.\left.-B^{2} \frac{\partial}{\partial \theta} R^{2}\right)+\frac{|\nabla \psi|^{2} q^{\prime}}{B^{2}}-\frac{R_{0} \Phi^{\prime}\left(g p^{\prime}+g^{\prime} B^{2}\right)}{\mathcal{F}^{2} \pi B_{0} B^{3}}\right], \\
& \kappa_{6}=\frac{B_{0} \mathcal{F} \Phi^{\prime}}{B \mathcal{J} \pi}\left[\frac{q^{\prime}}{B^{2}} \frac{\partial}{\partial \theta}|\nabla \psi|^{2}-\frac{|\nabla \psi|^{2} q^{\prime}}{B^{4} R^{2}}\left(B_{0}^{2} \mathcal{F}^{2} \frac{\partial}{\partial \theta}|\nabla \psi|^{2}-B^{2} \frac{\partial}{\partial \theta} R^{2}\right)\right], \\
& \frac{\partial}{\partial \psi} \frac{|\nabla \Phi|^{2}}{B^{2}}=\frac{|\nabla \psi|^{2}}{B^{2}}\left(2 \Phi^{\prime} \Phi^{\prime \prime}\right)+\frac{\left(\Phi^{\prime}\right)^{2}}{B^{2}} \frac{\partial}{\partial \psi}|\nabla \psi|^{2}-\frac{\left(\Phi^{\prime}\right)^{2}|\nabla \psi|^{2}}{B^{4}} \\
& \times\left[\frac{B_{0}^{2}}{R^{2}}\left(2 \mathcal{F} \mathcal{F}^{\prime}|\nabla \psi|^{2}+\mathcal{F}^{2} \frac{\partial}{\partial \psi}|\nabla \psi|^{2}+2 g g^{\prime} R_{0}^{2}\right)-\frac{B^{2}}{R^{2}} \frac{\partial}{\partial \psi} R^{2}\right],
\end{aligned}
$$

and

$$
\frac{\partial}{\partial \psi}|\nabla \psi|^{2}=-\frac{\mathcal{F}^{\prime}}{\mathcal{F}}|\nabla \psi|^{2}-\frac{\partial}{\partial \theta} \nabla \psi \cdot \nabla \theta-\frac{R^{2}}{B_{0}^{2} \mathcal{F}^{2}}\left(p^{\prime}+B_{0}^{2} R_{0}^{2} \frac{g g^{\prime}}{R^{2}}\right) .
$$

We define a scaled Jacobian $\hat{\mathcal{J}} \equiv \mathcal{J} / B_{0} \mathcal{F}$ such that $\mathbf{b} \cdot \nabla=(1 / B \hat{\mathcal{J}})(\partial / \partial \theta)$, acting only on $\hat{\phi}(\theta)$, so that our linearized gyrokinetic equation (2) becomes 


$$
\begin{gathered}
\frac{v_{\|}(\theta)}{\hat{\mathcal{J}}(\theta) B(\theta)} \frac{\partial}{\partial \theta} \hat{h}_{\sigma j}-i\left[\omega+\omega_{1}+i \nu_{f j}-\omega_{d j}(\theta)\right] \hat{h}_{\sigma j}(\theta)=-i\left\{\omega+\omega_{1}+\sigma \omega_{2}(\theta)+i u_{\zeta j} \mathbf{b} \cdot \nabla-\omega_{* j}\right. \\
\left.\times\left[1+\eta_{j}\left(\frac{E}{T_{j}}-\frac{3}{2}\right)+2 \frac{r_{n j}}{r_{u \zeta j}} \hat{u}_{\zeta j}\left(\hat{v}_{\|}-\hat{u}_{\zeta j}\right)\right]\right\} \frac{e_{j}}{T_{j}} F_{j} \hat{\phi}(\theta) J_{0}(\theta) .
\end{gathered}
$$

where $\omega_{1} \equiv n \Phi^{\prime} / B_{0} \mathcal{F}$ is the Doppler shift frequency and $\omega_{2}(\theta) \equiv\left(n m_{j} c / B_{0}^{2} \mathcal{F} e_{j}\right)\left(\Phi^{\prime \prime} / \mathcal{F}-\right.$ $\left.\Phi^{\prime} \mathcal{F}^{\prime} / \mathcal{F}^{2}\right)\left|R v_{\|}-\left(R v_{\|}\right)^{(0)}\right| \propto \omega_{E}$. Note that $\omega_{d j}$ as given by Eq. (3) contains both even terms and odd terms in $\sigma \equiv \operatorname{sign}\left(v_{\|}\right)$, unlike the usual magnetic drift frequency, which contains only even terms; we separate $\omega_{d j}$ into even and odd parts, $\omega_{d j}=\omega_{d j}^{e}+\sigma \omega_{d j}^{o}$. Then, we can solve Eq. (12) for $\hat{h}_{\sigma j}$ by a straightforward extension of the method of Ref. 3. The solution for circulating particles is the same as that in Ref. 3 with the substitution of $\left\{\omega+\omega_{1}+\sigma \omega_{2}(\theta)+i u_{\zeta j} \mathbf{b} \cdot \nabla-\omega_{* j}\left[1+\eta_{j}\left(E / T_{j}-3 / 2\right)+2\left(r_{n j} / r_{u \zeta j}\right) \hat{u}_{\zeta j}\left(\hat{v}_{\|}-\hat{u}_{\zeta j}\right)\right]\right\}$ for $\left(\omega-\omega_{* j}^{T}\right)$. The trapped-particle solution is the same as that in Ref. 3, with the corresponding substitution, and with additional terms due to $\omega_{d j}^{o}$ :

$$
\begin{aligned}
\hat{h}_{\sigma j}(\theta)= & \frac{e_{j}}{T_{j}} F_{j}\left\{\omega+\omega_{1}+i u_{\zeta, j} \mathbf{b} \cdot \nabla-\omega_{* j}\left[1+\eta_{j}\left(E / T_{j}-3 / 2\right)+2\left(r_{n j} / r_{u \zeta j}\right) \hat{u}_{\zeta j}\right.\right. \\
& \left.\left.\times\left(\hat{v}_{\|}-\hat{u}_{\zeta, j}\right)\right]\right\} \omega_{b}^{-1} \sum_{p=-\infty}^{\infty}\left[\frac{\Psi_{\sigma}^{p} \exp \left[i p \hat{\theta}(\theta)+i \sigma w_{d}^{e}(\theta)+i w_{d}^{o}(\theta)\right]}{\Gamma^{e}+\sigma \Gamma^{o}-\sigma p}+\frac{\sigma(-1)^{p}}{2}\right. \\
& \times \exp \left\{i \sigma\left[\Gamma^{e} \hat{\theta}(\theta)+w_{d}^{e}(\theta)\right]+i\left[\Gamma^{o} \hat{\theta}(\theta)+w_{d}^{o}(\theta)\right]\right\}\left(\frac{\cos \pi \Gamma^{o}}{\cos \pi \Gamma^{e}}-\sigma \frac{\sin \pi \Gamma^{o}}{\sin \pi \Gamma^{e}}\right) \\
& \left.\times\left(\frac{\Psi_{+}^{p}}{p-\Gamma^{e}-\Gamma^{o}}+\frac{\Psi_{-}^{p}}{p+\Gamma^{e}-\Gamma^{o}}\right)\right] \\
& +\frac{e_{j}}{T_{j}} F_{j} \sigma \sum_{p=-\infty}^{\infty}\left[\frac{\Psi_{2 \sigma}^{p} \exp \left[i p \hat{\theta}(\theta)+i \sigma w_{d}^{e}(\theta)+i w_{d}^{o}(\theta)\right]}{\Gamma^{e}+\sigma \Gamma^{o}-\sigma p}+\frac{\sigma(-1)^{p}}{2}\right. \\
& \times \exp \left\{i \sigma\left[\Gamma^{e} \hat{\theta}(\theta)+w_{d}^{e}(\theta)\right]+i\left[\Gamma^{o} \hat{\theta}(\theta)+w_{d}^{o}(\theta)\right]\right\}\left(\frac{\cos \pi \Gamma^{o}}{\cos \pi \Gamma^{e}}-\sigma \frac{\sin \pi \Gamma^{o}}{\sin \pi \Gamma^{e}}\right) \\
& \left.\times\left(\frac{\Psi_{2+}^{p}}{p-\Gamma^{e}-\Gamma^{o}}+\frac{\Psi_{2-}^{p}}{p+\Gamma^{e}-\Gamma^{o}}\right)\right]
\end{aligned}
$$

so that

$$
\begin{aligned}
\hat{h}_{+j}(\theta) \pm \hat{h}_{-j}(\theta)= & \frac{e_{j}}{T_{j}} F_{j}\left\{\omega+\omega_{1}+i u_{\zeta j} \mathbf{b} \cdot \nabla-\omega_{* j}\left[1+\eta_{j}\left(E / T_{j}-3 / 2\right)+2\left(r_{n j} / r_{u \zeta j}\right)\right.\right. \\
& \left.\left.\times \hat{u}_{\zeta j}\left(\hat{v}_{\|}-\hat{u}_{\zeta j}\right)\right]\right\} \omega_{b}^{-1} \sum_{p=-\infty}^{\infty}\left[\operatorname { e x p } [ i p \hat { \theta } ( \theta ) + i w _ { d } ^ { o } ( \theta ) ] \left(\frac{\Psi_{+}^{p} \exp \left[i w_{d}^{e}(\theta)\right]}{\Gamma^{e}+\Gamma^{o}-p}\right.\right. \\
& \left. \pm \frac{\Psi_{-}^{p} \exp \left[-i w_{d}^{e}(\theta)\right]}{\Gamma^{e}-\Gamma^{o}+p}\right)-(-1)^{p} \exp \left\{i\left[\Gamma^{o}+w_{d}^{o}(\theta)\right]\right\}\left(\frac{\cos \pi \Gamma^{o}}{\cos \pi \Gamma^{e}}\right.
\end{aligned}
$$




$$
\begin{aligned}
& \left.\times\left\{\begin{array}{c}
\sin \\
\cos
\end{array}\right\}\left[\Gamma^{e} \hat{\theta}(\theta)+w_{d}^{e}(\theta)\right]-\frac{\sin \pi \Gamma^{o}}{\sin \pi \Gamma^{e}}\left\{\begin{array}{c}
\cos \\
i \sin
\end{array}\right\}\left[\Gamma^{e} \hat{\theta}(\theta)+w_{d}^{e}(\theta)\right]\right) \\
& \left.\times\left(\frac{\Psi_{+}^{p}}{\Gamma^{e}+\Gamma^{o}-p}-\frac{\Psi_{-}^{p}}{\Gamma^{e}-\Gamma^{o}+p}\right)\right]+\frac{e_{j}}{T_{j}} F_{j} \sum_{p=-\infty}^{\infty} \\
& \times\left[\exp \left[i p \hat{\theta}(\theta)+i w_{d}^{o}(\theta)\right]\left(\frac{\Psi_{2+}^{p} \exp \left[i w_{d}^{e}(\theta)\right]}{\Gamma^{e}+\Gamma^{o}-p} \mp \frac{\Psi_{2-}^{p} \exp \left[-i w_{d}^{e}(\theta)\right]}{\Gamma^{e}-\Gamma^{o}+p}\right)\right. \\
& -(-1)^{p} \exp \left\{i\left[\Gamma^{o}+w_{d}^{o}(\theta)\right]\right\}\left(\frac { \operatorname { c o s } \pi \Gamma ^ { o } } { \operatorname { c o s } \pi \Gamma ^ { e } } \left\{\operatorname { c o s } \left\{\left[\Gamma^{e} \hat{\theta}(\theta)+w_{d}^{e}(\theta)\right]\right.\right.\right. \\
& i \sin \} \\
& -\frac{\sin \pi \Gamma^{o}}{\sin \pi \Gamma^{e}}\left\{i \sin \left\{\left[\Gamma^{e} \hat{\theta}(\theta)+w_{d}^{e}(\theta)\right]\right)\left(\frac{\Psi_{2+}^{p}}{\Gamma^{e}+\Gamma^{o}-p}-\frac{\Psi_{2-}^{p}}{\Gamma^{e}-\Gamma^{o}+p}\right)\right]
\end{aligned}
$$

where $\Gamma^{e} \equiv\left(\omega+i \nu_{f j}-\omega_{d j}^{e(0)}\right) / \omega_{b}, \Gamma^{o} \equiv-\omega_{d j}^{o(0)} / \omega_{b}, w_{d}^{e}(\theta) \equiv-\int_{\theta_{1}}^{\theta} d \theta^{\prime \prime}\left[\omega_{d j}^{e}\left(\theta^{\prime \prime}\right)-\right.$ $\left.\omega_{d j}^{e(0)}\right] B \hat{\mathcal{J}} /\left|v_{\|}\left(\theta^{\prime \prime}\right)\right|, w_{d}^{o}(\theta) \equiv-\int_{\theta_{1}}^{\theta} d \theta^{\prime \prime}\left[\omega_{d j}^{o}\left(\theta^{\prime \prime}\right)-\omega_{d j}^{o(0)}\right] B \hat{\mathcal{J}} /\left|v_{\|}\left(\theta^{\prime \prime}\right)\right|, \Psi_{2 \sigma}^{p}$ is the same as $\Psi_{\sigma}^{p}$ with an extra factor of $\omega_{2}(\theta) / \omega_{b}$ in the integrand, and the rest of the notation is the same as in Ref. 4. Employing the results for $\hat{h}_{\sigma j}(\theta)$ in the quasineutrality condition gives us the eigenmode integral equation. The perturbed electrostatic potential $\hat{\phi}(\theta)$ is then expanded in terms of chosen basis functions (Hermite functions) so that the integral equation is converted into a matrix equation, which is solved by standard methods. The solution procedure, and the electromagnetic generalization, are described in detail in Refs. 3 and 4.

\section{LINEAR ROTATION RESULTS}

To investigate the effects of rotation, we start with a case that was investigated in Ref. 8 using the old " $V_{\phi}$ " rotation model of Refs. 5 and 6 , and recalculate the linear growth rates $\gamma$ and the real frequencies $\omega_{r}$ using our new " $E_{r}$ " rotation model as described in the previous section. This is a case with experimentally-derived density and temperature profiles and a numerically-calculated MHD equilibrium for the TFTR "enhanced reversed shear" (ERS) discharge 84011 , at $t=2.70 \mathrm{~s}$, just before the ERS confinement transition time. We do the calculation for the electrostatic toroidal drift mode, including a carbon impurity species and 
a hot beam species with a slowing-down equilibrium distribution function. For this case we will use the $E_{r}$ profile obtained from $E_{r}=V_{\phi} B_{\theta}-V_{\theta} B_{\phi}+\left(1 / e_{C} n_{C}\right) d p_{C} / d r$, with all the quantities on the right hand side being experimentally determined, except that for $V_{\theta}$ we use $V_{\theta}^{n e o}$, the neoclassical estimate ${ }^{26}$ for $V_{\theta}$. We define the local Mach number $M \equiv\left|v_{E}\right| / v_{i}$, and we will vary $M$ artificially by multiplying the entire $\Phi$ profile by a constant, and we will multiply the $V_{\phi}$ profile by the same constant.

Results for $\gamma$ and $\omega_{r}$ versus $M$ for $r / a=0.2$ for this TFTR ERS case with $n=21$, $k_{\theta} \rho_{i}=0.88$, and ballooning parameter ${ }^{22} \theta_{0}=0$ are shown in Figs. $1 \mathrm{a}$ and $1 \mathrm{~b}$, respectively. Curve (i) shows the effect of this artificial variation of $M$, keeping the first and second terms in our Taylor series expansion of $\mathbf{k} \cdot \mathbf{u}_{E}$ [the $\omega_{1}$ and $\omega_{2}(\theta)$ terms in Eq. (12) and the last term in $\omega_{d j}(\theta)$ in Eq. (3)] as the only rotational terms. We see that these terms can decrease the growth rate substantially, but do not give complete stabilization at any relevant value of $M$. For curve(ii), we add in the rest of the terms for the $E_{r}$ rotation model [the $\kappa_{3}, \kappa_{4}, \kappa_{5}$, and $\kappa_{6}$ terms in $\omega_{d j}(\theta)$ in Eq. (3)]; we see that the mode can be almost completely stabilized, but only at values of $M$ that are several times higher than the experimental value. Curve (iii) shows the corresponding results obtained with the old $V_{\phi}$ model, and these are seen to be roughly similar to those with the new $E_{r}$ model, at least in this case, despite the differences in the two models.

Another point to notice in Fig. 1 is that the experimental value of $M$ falls short by a factor of several from that needed to completely stabilize the mode. Also, the needed $\mathbf{E} \times \mathbf{B}$ level for linear stabilization from the heuristic stabilization criterion $\omega_{E} \sim \gamma_{0}$, observed in ITG mode simulations, ${ }^{14,16}$ where $\gamma_{0}$ is the linear growth rate without rotation effects, would say that the mode should not be stabilized here, because $\left(\omega_{E}\right)^{\exp }$ falls short of $\gamma_{0}$ by a factor of several also. The two shortfall factors differ by a factor of two or more, but we have at least rough qualitative agreement between the two stabilization criteria, if $E_{r}$ were to be scaled up to those levels. However, the $E_{r}$ rotation model variation of $\gamma$ with $M$ is not linear for this case! The results from the $E_{r}$ model in general show both linear and quadratic behavior, as well as 'flat spots' and 'tails', for different cases in different ranges of $M$. 

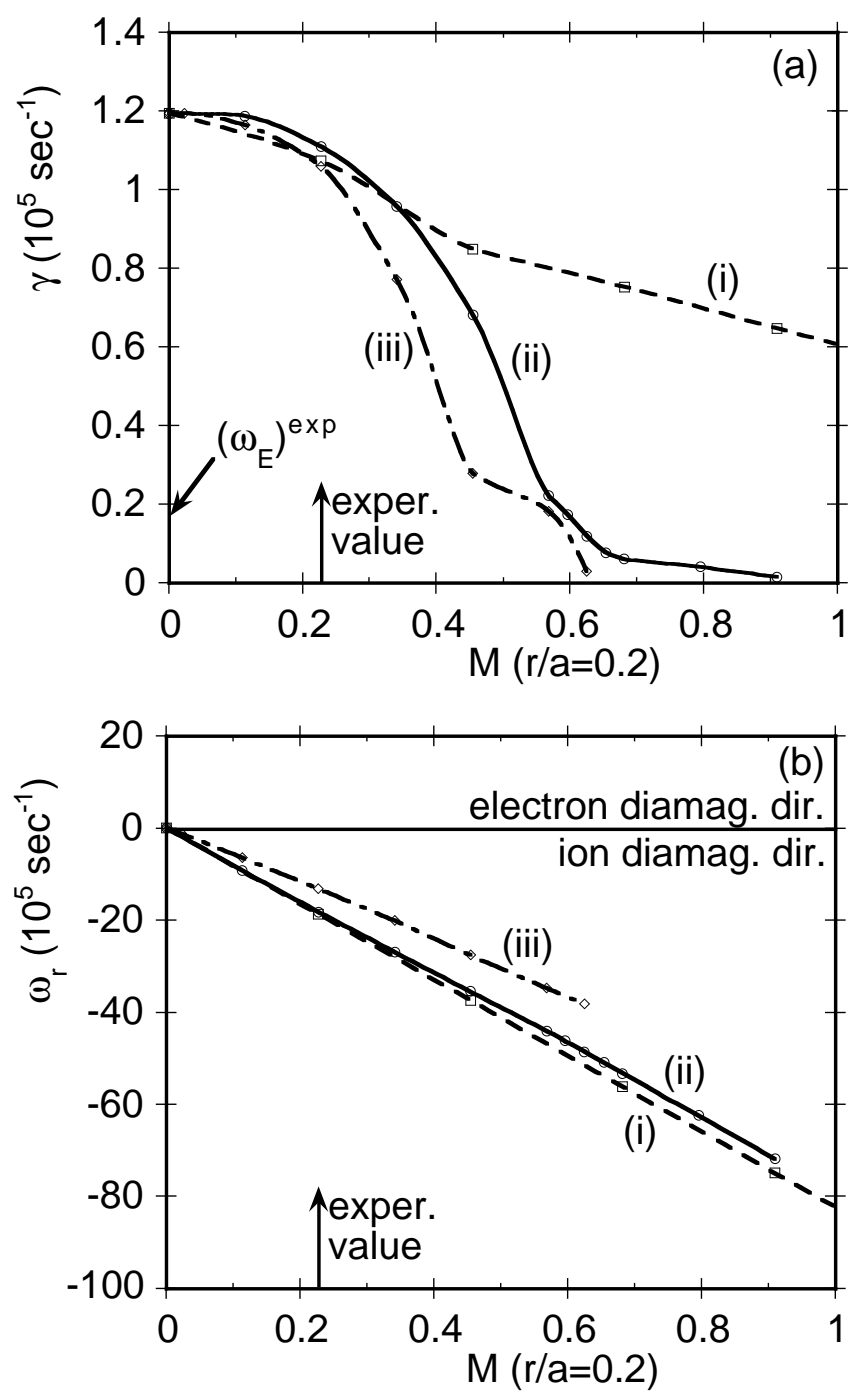

FIG. 1. (a) Linear growth rate $\gamma$ and (b) real frequency $\omega_{r}$ for TFTR ERS discharge 84011 at $t=2.70 \mathrm{~s}$, for the electrostatic toroidal drift mode with carbon and a slowing-down beam, for $r / a=0.200, n=21, k_{\theta} \rho_{i}=0.88$, and $V_{\phi} \propto M$. Here, curve (i) corresponds to the $E_{r}$ rotation model with the $\omega_{1}$ and $\omega_{2}(\theta)$ terms only, curve (ii) to the $E_{r}$ model with all terms, and curve (iii) to the $V_{\phi}$ rotation model. 
The corresponding results for this TFTR ERS case for $r / a=0.3$ are shown in Fig. 2 for $n=41$ and $k_{\theta} \rho_{i}=0.79$. The linear growth rates in Fig. 2 a are shown in curve (i) with only the first and second Taylor series terms as the rotational terms, and again these are only partially stabilizing. With all the terms in the $E_{r}$ model, as shown in curve (ii), $\gamma$ is almost linear in $M$, with one 'flat spot'. Curve (iii), for the old $V_{\phi}$ model, is at first almost flat and then quadratic as $M$ increases, whereas curve(ii) for the $E_{r}$ model is almost linear (with a 'flat spot'), but the corresponding $M_{c r i t}$ values for marginal stability are almost the same. Also, for this radius, $\gamma_{0} /\left(\omega_{E}\right)^{\exp }$ is almost exactly equal to $M_{c r i t} / M_{\exp }$. In this sense, the $E_{r}$ rotation model and the heuristic criterion for stabilization $\omega_{E} \sim \gamma_{0}$ are in nearly exact agreement for this case, if the $E_{r}$ level were to be scaled up. The real frequencies, shown in Fig. 2b, are again almost linear, as expected.

Another TFTR ERS case of interest is for discharge 103794, at $t=2.0 \mathrm{~s}$, which is the time when the measured value of $V_{\theta}$ reaches its maximum magnitude. We will compare three different sets of input data for the $E_{r}$ radial profile: $E_{r}=0$, corresponding to an absence of rotation, $E_{r}=E_{r}\left(V_{\theta}^{n e o}\right)$, where the neoclassical estimate ${ }^{26}$ of $V_{\theta}$ is used to calculate $E_{r}$ from the radial force balance relation $E_{r}=V_{\phi} B_{\theta}-V_{\theta} B_{\phi}+\left(1 / e_{C} n_{C}\right) d p_{C} / d r$, and $E_{r}=E_{r}\left(V_{\theta}^{\text {meas }}\right)$ where the spectroscopically measured profile ${ }^{27}$ of $V_{\theta}$ is used in the same relation. The radial profiles for $V_{\theta}^{\text {neo }}$ and $V_{\theta}^{\text {meas }}$ can be substantially different, by an order of magnitude or more, at localized times and places in the discharge. Correspondingly, the associated profiles for $E_{r}$, shown in Fig. 3, and of the radial derivative of $E_{r}$, are drastically different in the inner half of the cross-section, though roughly comparable in the outer half. The corresponding results for the linear growth rates of the electrostatic toroidal drift mode, for $k_{\theta} \rho_{i}=0.81$ and $\theta_{0}=0$, are shown in Fig. 4 for the three choices for $E_{r}$. The mode is stable for $r / a \simeq 0.3$ due to a flat spot there in the pressure profile. For $r / a>0.3$, the (outer) growth rate profiles are almost identical for $E_{r}=0$ and for $E_{r}\left(V_{\theta}^{n e o}\right)$, while the growth rate profile for $E_{r}\left(V_{\theta}^{\text {meas }}\right)$ is somewhat narrowed and is lowered by $\sim 30 \%$. For $0.1<r / a<0.3$, the mode is unstable for $E_{r}=0$, but this (inner) growth rate profile is lowered by $\sim 40 \%$ and narrowed to $0.2<r / a<0.3$ for $E_{r}\left(V_{\theta}^{n e o}\right)$. However, for $E_{r}=E_{r}\left(V_{\theta}^{\text {meas }}\right)$, the mode is completely 

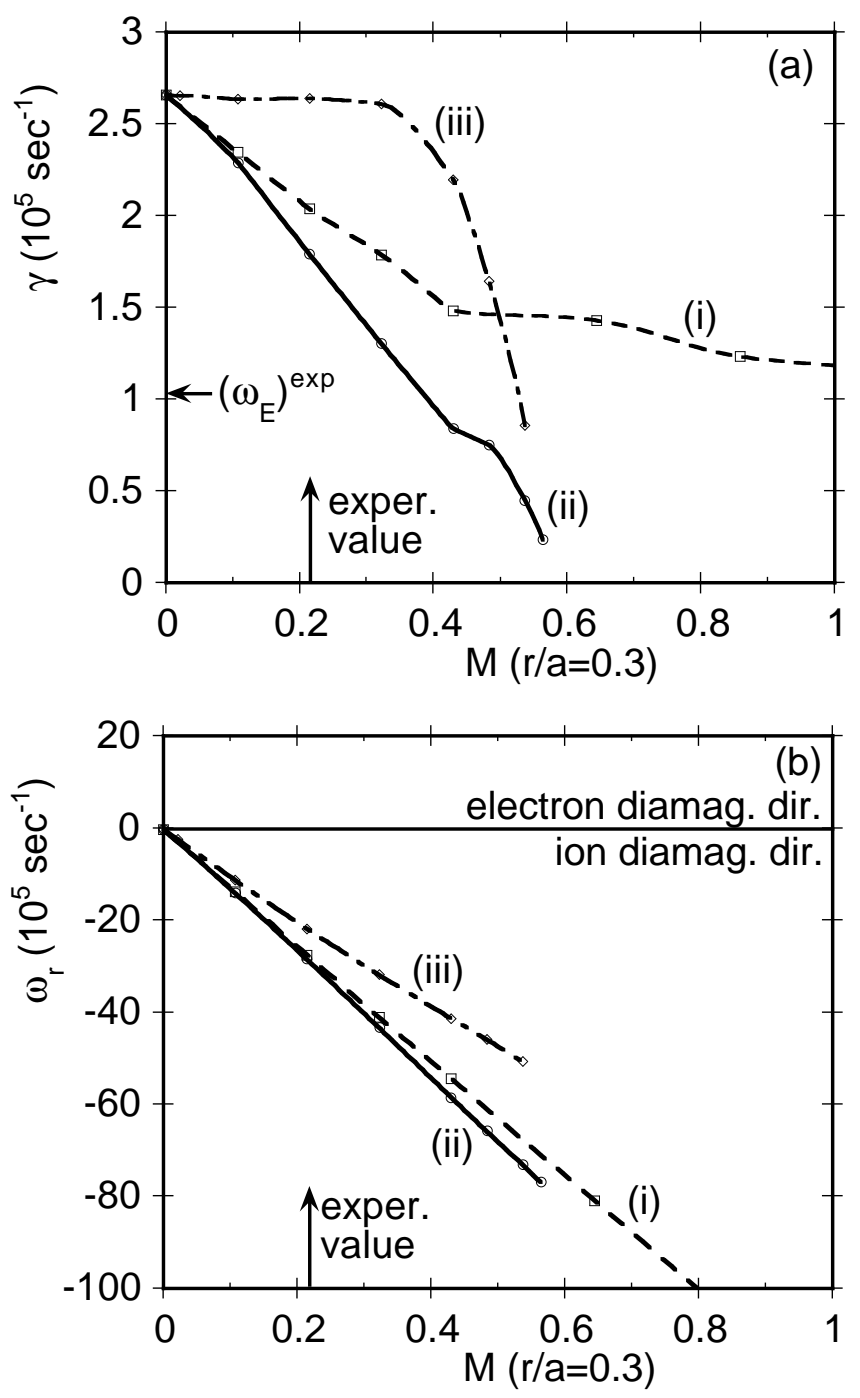

FIG. 2. As in Fig. 1, except with $r / a=0.300, n=41$, and $k_{\theta} \rho_{i}=0.79$. 
stabilized in this inner region. In fact, even for $E_{r}=0.1 \times E_{r}\left(V_{\theta}^{\text {meas }}\right)$, the mode would still be completely stabilized, so that it is likely that this linear stabilization would actually occur at an earlier time, when $V_{\theta}^{\text {meas }}$ is not as large.

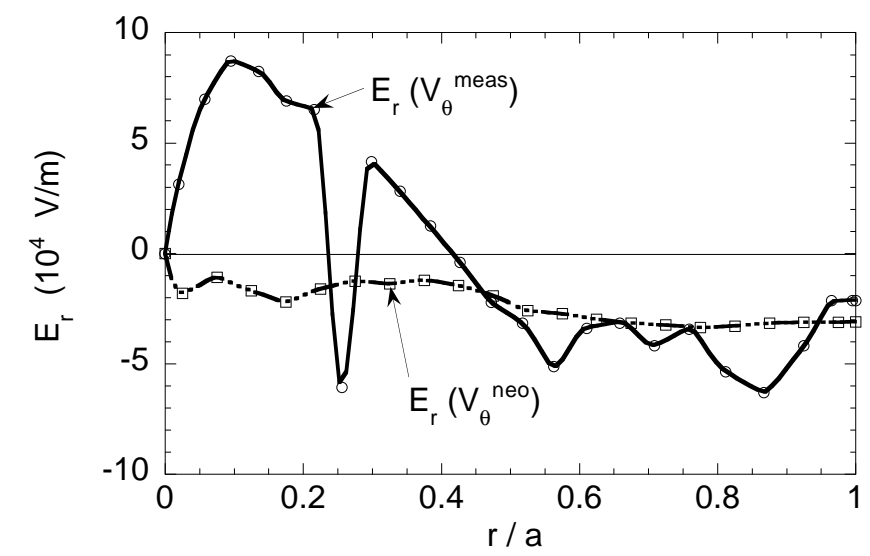

FIG. 3. Radial profiles of $E_{r}\left(V_{\theta}^{n e o}\right)$ and $E_{r}\left(V_{\theta}^{\text {meas }}\right)$ for TFTR ERS discharge 103794 at $t=2.0 \mathrm{~s}$.

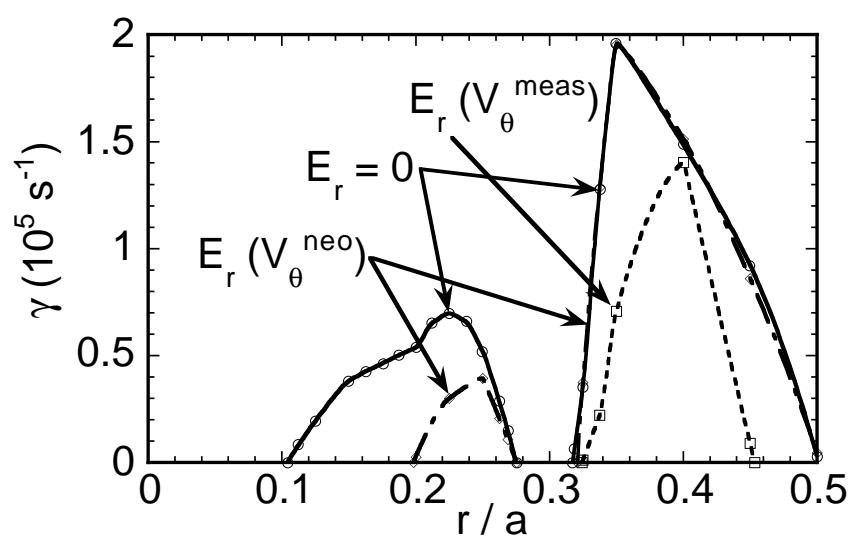

FIG. 4. Radial profile for $\gamma$ for TFTR ERS discharge 103794 at $t=2.0 \mathrm{~s}$, for the electrostatic toroidal drift mode with carbon and a slowing-down beam, with $E_{r}=0, E_{r}\left(V_{\theta}^{\text {neo }}\right)$, and $E_{r}\left(V_{\theta}^{\text {meas }}\right)$.

The corresponding experimental profile for $\omega_{E}\left(V_{\theta}^{\text {meas }}\right)$ is shown in Fig. 5, along with the growth rate curves from Fig. 4 . Comparing the $\omega_{E}\left(V_{\theta}^{\text {meas }}\right)$ curve with the $E_{r}=0$ growth rate curve, which is the curve for the growth rate $\gamma_{0}$ without rotation, the heuristic criterion would say that for the inner region $0.15<r / a<0.3$ the mode should be stable, since $\omega_{E}>\gamma_{0}$ there, and the result with the new rotation model using $E_{r}\left(V_{\theta}^{\text {meas }}\right)$ also is that the 
mode is stable there. For $0.34<r / a<0.42, \omega_{E}<\gamma_{0}$, so the heuristic criterion says that the mode should be unstable in that region. In fact, the new rotation model calculation with $E_{r}\left(V_{\theta}^{\text {meas }}\right)$ gives a slightly wider region of instability, $0.32<r / a<0.45$, but this extent is reasonably close. Thus, the FULL code calculation with the $E_{r}$ rotation model can give comparable results to the heuristic criterion, though we saw for the previous cases that they are often similar only within a factor of two or so on the necessary stabilizing level of $E_{r}$. Once linear stabilization is achieved in this inner region, so that the anomalous transport is suppressed there, nonlinear processes of the sort described by Diamond, et al. ${ }^{28}$ and Newman, et al. ${ }^{29}$ and others can act so as propagate this region of suppression outward.

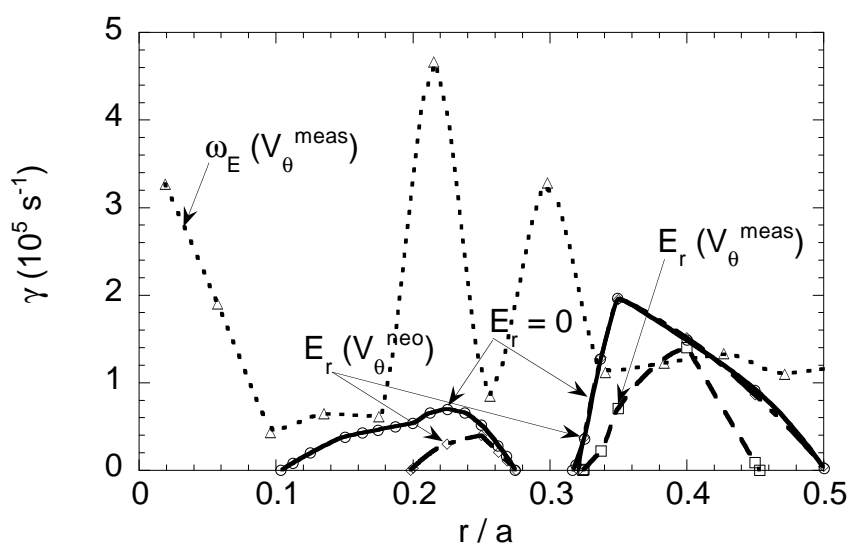

FIG. 5. As in Fig. 4, but with a different vertical scale, and showing also $\omega_{E}\left(V_{\theta}^{\text {meas }}\right)$.

\section{STUDY OF FLUCTUATION-GENERATED FLOW IN GYROKINETIC SIMULATIONS}

Recent results from TFTR,${ }^{27}$ of the sort shown in Fig. 3, indicate that significant, radially localized $\mathbf{E} \times \mathbf{B}$ flow can be generated in the core by some mechanism which cannot be described by the present neoclassical theory. While there is no quantitative theoretical prediction to date which can be compared to the experimental data directly, it has been observed in many simulations that fluctuations can generate $\mathbf{E} \times \mathbf{B}$ flow. In particular, the dominant role of the fluctuation-generated small scale $\mathbf{E} \times \mathbf{B}$ flow in the nonlinear satura- 
tion of toroidal ITG turbulence, ${ }^{30,13,14}$ including the case with trapped electron dynamics, ${ }^{15}$ has been observed in gyrofluid flux-tube simulations. Its importance has been also observed in gyrofluid simulations in sheared slab geometry, ${ }^{31}$ and in gyrokinetic flux-tube ${ }^{32}$ and annulus ${ }^{19}$ simulations. It is therefore of vital importance for realistic nonlinear simulations to accurately treat the dynamics of the fluctuation-generated $\mathbf{E} \times \mathbf{B}$ flow, as emphasized in Ref. 33.

We also note that such flows with wider radial scales on the order of a fraction of the system size have been often observed in previous nonlinear simulations. These include particle simulations of electron drift waves, ${ }^{34}$ gyrokinetic simulations of ITG instabilities ${ }^{35}$ in slab geometry, fluid simulations of dissipative drift wave turbulence models, ${ }^{36}$ and fluid simulations of pressure gradient driven turbulence. ${ }^{37}$ Theoretical discussions regarding the flow generation mechanism via Reynolds' stress and its potential importance in enhancing confinement can be found in Ref. 38. A simple dynamic model based on this mechanism indicates that the poloidal flow shear excursion is usually of limited duration. ${ }^{39}$ Finally, there is experimental evidence of fluctuation-generated small scale $\mathbf{E} \times \mathbf{B}$ flow from the DIII-D edge as well. ${ }^{40}$

A new toroidal gyrokinetic particle code has been developed using magnetic coordinates and Hamiltonian guiding center equations of motion. By utilizing a non-spectral Poisson solver, ${ }^{41}$ the new turbulence code can efficiently handle general geometry and realistic equilibrium profiles. The dynamics of fluctuation-driven poloidal $\mathbf{E} \times \mathbf{B}$ flow is studied in this section using both global and annular simulations with a variety of boundary conditions. The residual poloidal flow in response to an initial perturbation which is constant on a magnetic surface has been calculated by an initial value approach. ${ }^{33}$ Here, we solve the following gyrokinetic equation with an initial source $\delta f_{00}$ through gyrokinetic simulations,

$$
\left[\frac{\partial}{\partial t}+\left(v_{\|} \hat{\mathbf{b}}+\mathbf{v}_{d}+\mathbf{u}_{E}\right) \cdot \frac{\partial}{\partial \mathbf{x}}-\hat{\mathbf{b}}^{*} \cdot \nabla(\mu B+\Phi) \frac{\partial}{\partial v_{\|}}\right] f=\delta f_{00} \delta(t),
$$

where $\hat{\mathbf{b}}^{*}=\hat{\mathbf{b}}+\left(v_{\|} / \Omega\right) \hat{\mathbf{b}} \times(\hat{\mathbf{b}} \cdot \nabla \hat{\mathbf{b}})$. In the simulations, the poloidal flow is initialized at $t=0$ and its steady state value is measured after a few bounce times. The residual levels of 
poloidal flow in the simulations, as shown in Fig. 6, agree well with the theoretical results of Ref. 33 in the high aspect ratio limit where the theory is valid. We note that similar results have also been obtained in flux-tube gyrokinetic simulations. ${ }^{42}$

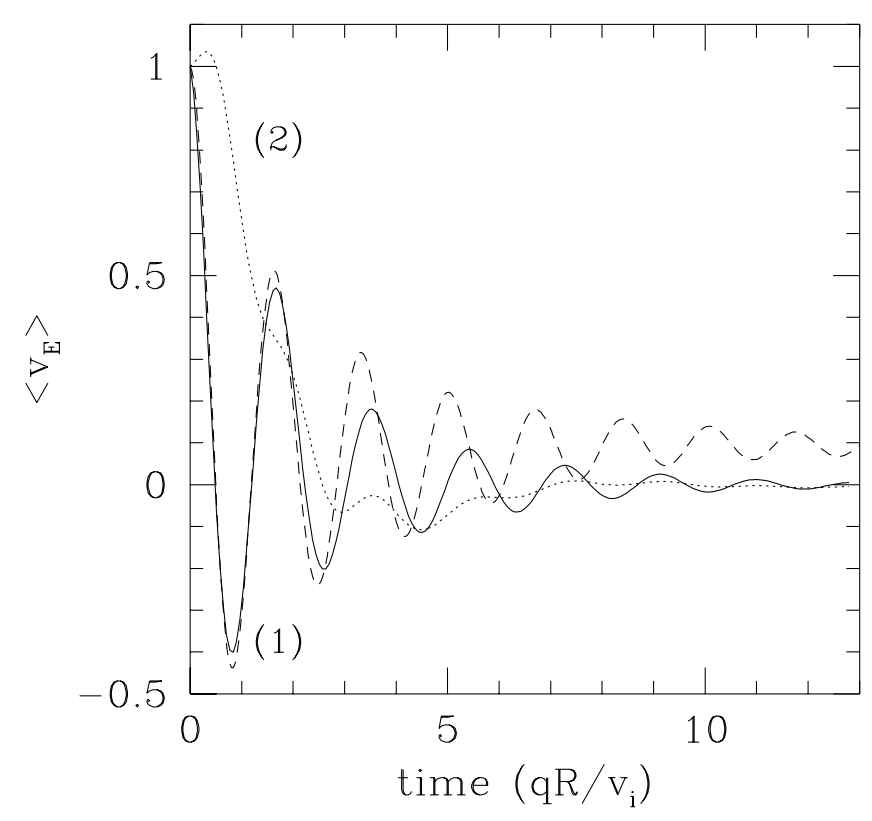

FIG. 6. Linear flow damping for gyrofluid (solid) and gyrokinetic (dashed) simulations for (1) initializing perpendicular flow only, $\Phi(r)$. Also shown are gyrofluid results (2) initializing perpendicular flow with a small parallel flow (dotted).

The relaxation of the initial poloidal flow in Fig. 6 occurs via damped oscillations with a characteristic frequency corresponding to that of the geodesic acoustic mode (GAM). ${ }^{43}$ In these processes, the $m=1$ harmonic can be excited by toroidal coupling to the $m=0$ harmonic. To be consistent with the analytic theory, the $m \neq 0$ harmonics are suppressed in the simulations on the ground that they are Landau damped. However, when the safety factor $q$ is large, the resonant particles move out to the Maxwellian tails and the $m=1$ harmonic becomes undamped. As a result, the oscillations of residual poloidal flow persist.

Since the microturbulence in tokamaks typically has a ballooning structure, it is important to be able to model the generation of poloidal flows from $m=1$ sources. This coupling of $m=1$ sources to the $m=0$ harmonic is predominantly due to trapped particle effects. 
Because of the up-down symmetry of the equilibrium magnetic field, only the part of the $m=1$ harmonic that is even in $\theta$ can generate poloidal flow. This has been verified by the simulation results, which show that the time asymptotic poloidal flow is finite for a $\theta$-even source and zero for a $\theta$-odd source.

Gyrokinetic simulation results in this section show that there is a significant enhancement of $\mathbf{E} \times \mathbf{B}$ flow generation with a non-zero $m$ component of the drive. We also note that in a generally shaped, finite aspect ratio tokamak, the $\mathbf{E} \times \mathbf{B}$ shearing rate can also have strong $\theta$-dependence even when $\Phi$ is a flux function. ${ }^{10}$ In-out asymmetry of the fluctuation suppression behavior in DIII-D, measured via heterodyne microwave scattering, ${ }^{44}$ is in qualitative agreement with a prediction based on this observation. ${ }^{45}$

\section{DYNAMICS OF TURBULENCE DRIVEN FLUCTUATING $\mathrm{E} \times \mathrm{B}$ FLOWS FROM GYROFLUID SIMULATION}

In gyrofluid flux-tube simulations, fluctuations in the electrostatic potential are nonlinearly driven by the turbulence, leading to radially sheared poloidal $\mathbf{E} \times \mathbf{B}$ flows. These "radial" modes are roughly constant on a flux surface, but have small radial scales, on the order of the turbulent scale size.

The linear damping of these poloidal $\mathbf{E} \times \mathbf{B}$ flows was shown to be crucial and was investigated in Ref. 15. The nonlinear drive of the flows is balanced by linear damping and nonlinear damping. We first investigate the linear damping of the flows, by initializing a perturbation and solving the linearized gyrofluid equations ${ }^{46}$ forward in time. This flow will initially experience fast collisionless damping, as shown in Fig. 6 for $k_{r} \rho_{i} / \sqrt{2}=0.2$. Here, two different initial conditions are used: (1) perpendicular flow only, by initializing only $\Phi(r)$, and (2) perpendicular flow with a small parallel flow. Both initializations show a damping rate on the order of a few transit times. Results from gyrokinetic particle simulations are also shown for case (1), as discussed in Sec. IV. The gyrofluid and gyrokinetic results agree very well in the fast linear damping phase. Later in time, there is a smaller residual linearly 
undamped component, depending on the initial conditions, as emphasized by Rosenbluth and Hinton. ${ }^{33}$ The present gyrofluid equations do not recover this small residual flow component with much accuracy, as trapped particle effects are important. For this residual flow to be significant nonlinearly, the nonlinear damping of the flows would have to be weak, so that the linear details could dominate. From the time history of the $k_{r} \rho_{i} / \sqrt{2}=0.2$ component of the potential over the saturated phase of a nonlinear gyrofluid simulation, its correlation function can be obtained, as shown in Fig. 7. Since the correlation time of the flow is on the order of the fast linear damping rate, the flow does not have time to relax to the residual flow component before nonlinear effects break it up. This correlation time is also on the order of the turbulent decorrelation time of the turbulence. When the nonlinear effects are strong enough, as in this case, the short correlation time indicates that nonlinear damping is saturating this component. Also, modifying the gyrofluid equations to change the linear level of residual flow does not seem to change the heat flux as long as the fast linear damping is retained, and the turbulence is not very weak.

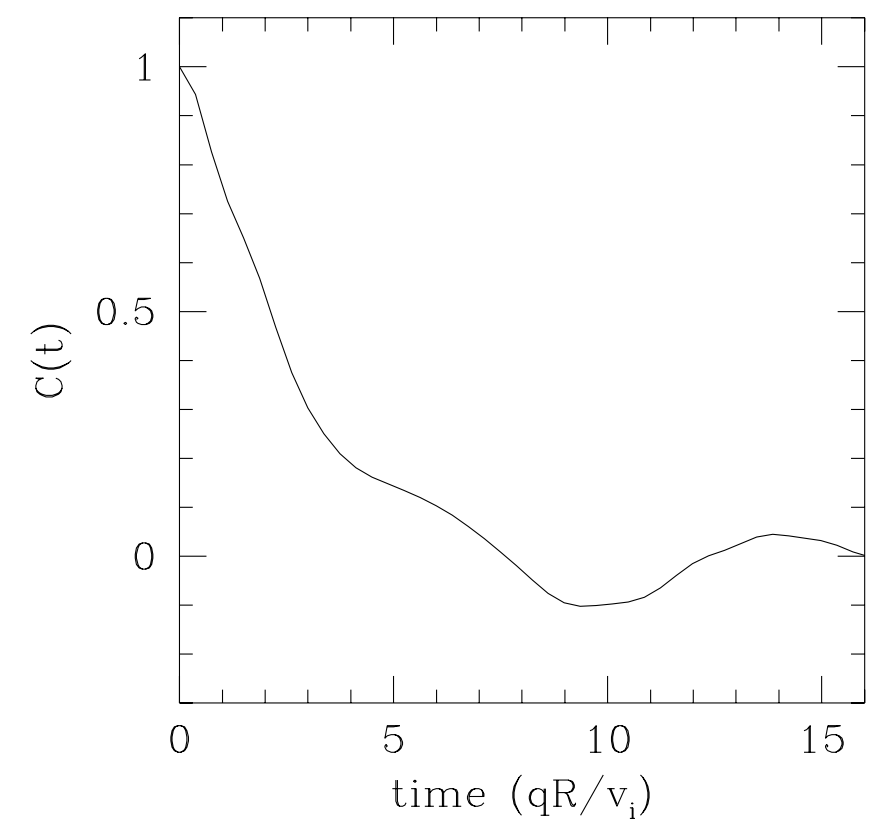

FIG. 7. Correlation function of the $k_{\theta} \rho_{i} / \sqrt{2}=0.2$ component of the flow from a nonlinear gyrofluid simulation. 


\section{CONCLUSIONS}

An improved linear rotation model and its implementation in the FULL code linear eigenfrequency-eigenfunction calculation have been described. This ' $E_{r}$ ' model is formulated in terms of the radial electric field, $E_{r}=V_{\phi} B_{\theta}-V_{\theta} B_{\phi}+\left(1 / e_{C} n_{C}\right) d p_{C} / d r$, and thus contains rotational contributions from $V_{\phi}, V_{\theta}$, and $d p / d r$, whereas the older ' $V_{\phi}$ ' rotation model only contains the contribution of $V_{\phi}$. Also, the new $E_{r}$ model includes terms proportional to the $\mathbf{E} \times \mathbf{B}$ shearing frequency (in flux coordinates) in the final mode equation. Several TFTR ERS cases were presented, in which the old and new rotation models were compared, and shown to give roughly comparable Mach numbers for complete stabilization of the electrostatic toroidal drift mode. Comparisons are also made with the heuristic criterion, $\omega_{E} \sim \gamma_{0}$, for complete stabilization, and the needed $E_{r}$ values are seen to be similar within factors of two or so. Finally, a comparison of results using the neoclassically estimated profile

of $V_{\theta}$ and the much larger experimentally measured profile show that $V_{\theta}^{\text {meas }}$ is sufficient to give complete stabilization in the inner unstable region of one TFTR ERS discharge, while $V_{\theta}^{n e o}$ is not. Of course, the sometimes bursty fluctuation level changes ${ }^{47}$ and the confinement transitions and their propagation in tokamaks such as TFTR are really nonlinear phenomena, but nonlinear studies such as those in Refs. 28 and 29 indicate that some localized weakening of the underlying linear instability is needed for initiation of the transition. Weakening due to negative magnetic shear and evolution of the density and temperature profiles was considered in Ref. 8. Here, the weakening effects of sheared $\mathbf{E} \times \mathbf{B}$ rotation have been investigated.

Analytic calculations ${ }^{33}$ of the residual flows have been substantiated by gyrokinetic simulations. These results show that the $\theta$ dependence of the turbulent source has a significant impact on the flow generation and evolution. Nonlinear gyrofluid simulations find that the correlation time of the flows is on the order of the fast linear damping time, indicating that nonlinear damping effects may be playing a significant role in the long time evolution of the flows, at least in the strongly turbulent parameter regimes that we have studied. The residual flow level may be more important in weakly turbulent regimes near marginal stability. 
Good agreement is found between the fast linear damping of poloidal flows in gyrofluid and gyrokinetic simulations.

\section{ACKNOWLEDGMENTS}

The authors would like to thank Dr. R. E. Bell and Dr. E. J. Synakowski for supplying the TFTR data. This work was supported by United States Department of Energy Contract No. DE-AC02-76-CHO-3073, and in part by the U.S. DOE Fusion Energy Postdoctoral Research Program administered by the Oak Ridge Institute for Science and Education. 


\section{REFERENCES}

${ }^{1}$ D. J. Grove and D. M. Meade, Nucl. Fusion 25, 1167 (1985).

${ }^{2}$ F. M. Levinton, M. C. Zarnstorff, S. H. Batha, M. Bell, R. E. Bell, R. V. Budny, C. Bush, Z. Chang, E. Fredrickson, A. Janos, J. Manickam, A. Ramsey, S. A. Sabbagh, G. L. Schmidt, E. J. Synakowski, and G. Taylor, Phys. Rev. Lett. 75, 4417 (1995).

${ }^{3}$ G. Rewoldt, W. M. Tang, and M. S. Chance, Phys. Fluids 25, 480 (1982).

${ }^{4}$ G. Rewoldt, W. M. Tang, and R. J. Hastie, Phys. Fluids 30, 807 (1987).

${ }^{5}$ M. Artun, Ph.D. Dissertation, Princeton University, 1994.

${ }^{6}$ M. Artun, W. M. Tang, and G. Rewoldt, Phys. Plasmas 2, 3384 (1995).

${ }^{7}$ G. Rewoldt, L. L. Lao, and W. M. Tang, Phys. Plasmas 3, 4074 (1996).

${ }^{8}$ G. Rewoldt, L. L. Lao, and W. M. Tang, Phys. Plasmas 4, 3293 (1997).

9 T. S. Hahm, Phys. Plasmas 3, 4658 (1996).

10 T. S. Hahm and K. H. Burrell, Phys. Plasmas 2, 1648 (1995).

${ }^{11}$ R. J. Fonck, G. Cosby, R. D. Durst, S. F. Paul, N. Bretz, S. Scott, E. Synakowski, and G. Taylor, Phys. Rev. Lett. 70, 3736 (1993).

${ }^{12}$ S. E. Parker, W. W. Lee, and R. A. Santoro, Phys. Rev. Lett. 71, 2042 (1993).

${ }^{13}$ G. W. Hammett, M. A. Beer, W. Dorland, S. C. Cowley, and S. A. Smith, Plasma Phys. Contr. Fusion 35, 973 (1993).

${ }^{14}$ R. E. Waltz, G. D. Kerbel, and J. Milovich, Phys. Plasmas 1, 2229 (1994).

${ }^{15}$ M. A. Beer, Ph.D. Dissertation, Princeton University, 1995.

${ }^{16}$ R. E. Waltz, G. D. Kerbel, J. Milovich, and G. W. Hammett, Phys. Plasmas 2, 2408 (1995). 
${ }^{17}$ K. H. Burrell, Phys. Plasmas 4, 1499 (1997)

${ }^{18}$ E. J. Synakowski, S. H. Batha, M. A. Beer, M. G. Bell, R. E. Bell, R. V. Budny, C. E. Bush, P. C. Efthimion, T. S. Hahm, G. W. Hammett, B. LeBlanc, F. Levinton, E. Mazzucato, H. Park, A. T. Ramsey, G. Schmidt, G. Rewoldt, S. D. Scott, G. Taylor, and M. C. Zarnstorff, Phys. Plasmas 4, 1736 (1997).

19 Zhihong Lin, W. W. Lee, W. M. Tang, and R. B. White, Bull. Am. Phys. Soc. 42, 1985 (1997).

${ }^{20}$ F. L. Waelbroeck and L. Chen, Phys. Fluids B 3, 601 (1991).

${ }^{21}$ J. B. Taylor, H. R. Wilson, and J. W. Connor, Plasma Phys. Controlled Fusion 38, 243 $(1996)$.

${ }^{22}$ E. A. Frieman, G. Rewoldt, W. M. Tang, and A. H. Glasser, Phys. Fluids 23, 1750 (1980).

${ }^{23}$ H. E. Mynick and S. E. Parker, Bull. Am. Phys. Soc. 41, 1359 (1996).

${ }^{24}$ J. Y. Kim, Y. Kishimoto, M. Wakatani, and T. Tajima, Phys. Plasmas 3, 3689 (1996).

${ }^{25}$ M. Kotschenreuther, G. Rewoldt, and W. M. Tang, Comp. Phys. Comm. 88, 128 (1995).

${ }^{26}$ W. A. Houlberg, K. C. Shaing, S. P. Hirshman, and M. C. Zarnstorff, Phys. Plasmas 4, $3230(1997)$.

${ }^{27}$ R. E. Bell, Bull. Am. Phys. Soc. 42, 1945 (1997).

${ }^{28}$ P. H. Diamond, V. B. Lebedev, D. E. Newman, B. A. Carreras, T. S. Hahm, W. M. Tang, G. Rewoldt, and K. Avinash, Phys. Rev. Lett. 78, 1472 (1997).

${ }^{29}$ D. E. Newman, B. A. Carreras, D. Lopez-Bruna, and P. H. Diamond, Bull. Am. Phys. Soc. 42, 2032 (1997).

${ }^{30}$ M. A. Beer, G. W. Hammett, W. Dorland, and S. C. Cowley, Bull. Am. Phys. Soc. 37, $1478(1992)$. 
${ }^{31}$ W. Dorland, G. W. Hammett, T. S. Hahm, and M. A. Beer, Bull. Am. Phys. Soc. 37, $1478(1992)$.

${ }^{32}$ A. M. Dimits, T. J. Williams, J. A. Byers, B. I. Cohen, Phys. Rev. Lett. 77, 71 (1996).

${ }^{33}$ M. N. Rosenbluth and F. L. Hinton, Phys. Rev. Lett. 80, 724 (1998).

${ }^{34}$ W. W. Lee, Y. Y. Kuo, and H. Okuda, Phys. Fluids 21, 617 (1978).

${ }^{35}$ W. W. Lee, W. M. Tang, and H. Okuda, Phys. Fluids 23, 2007 (1980).

${ }^{36}$ A. Hasegawa and M. Wakatani, Phys. Rev. Lett. 59, 1581 (1987).

${ }^{37}$ B. A. Carreras, V. E. Lynch, and L. Garcia, Phys. Fluids B 3, 1438 (1991).

${ }^{38}$ P. H. Diamond and Y. B. Kim, Phys. Fluids B 3, 1626 (1991).

${ }^{39}$ P. H. Diamond, V. B. Lebedev, D. E. Newman, and B. A. Carreras, Phys. Plasmas 2, $3685(1995)$.

${ }^{40}$ S. Coda, M. Porkolab, and K. H. Burrell, Proceedings of the 24th European Physical Society Conference on Controlled Fusion and Plasma Physics, Berchtesgaden, Germany, (European Physical Society, Petit-Lancy, Switzerland, 1997) abstract P3.053, p. 343.

${ }^{41}$ Z. Lin and W. W. Lee, Phys. Rev. E 52, 5646 (1995).

42 A. M. Dimits, personal communication (1997).

${ }^{43}$ N. Winsor, J. L. Johnson, and J. M. Dawson, Phys. Fluids 11, 2448 (1968).

${ }^{44}$ C. L. Rettig, K. H. Burrell, B. W. Stallard, G. R. McKee, G. M. Staebler, T. L. Rhodes, C. M. Greenfield, and W. A. Peebles, "Dynamics of Core Transport Barrier Formation and Expansion in the DIII-D Tokamak," to appear in Phys. Plasmas.

45 T. S. Hahm and K. H. Burrell, Plasma Phys. Control. Fusion 38, 1427 (1996).

${ }^{46}$ M. A. Beer and G. W. Hammett, Phys. Plasmas 3, 4046 (1996). 
${ }^{47}$ E. Mazzucato, S. H. Batha, M. Beer, M. Bell, R. E. Bell, R. V. Budny, C. Bush, T. S. Hahm, G. W. Hammett, F. M. Levinton, R. Nazikian, H. Park, G. Rewoldt, G. L. Schmidt, E. J. Synakowski, W. M. Tang, G. Taylor, and M. Zarnstorff, Phys. Rev. Lett. 77,3145 (1996). 